\title{
Asthma Attack Prediction based on Weather Factors
}

\author{
Eman Alharbi ${ }^{1}$, Manal Abdullah ${ }^{2}$ \\ ${ }^{1}$ Department of Information System, Faculty of Computing and Information Technology, King Abdulaziz University, Jeddah, \\ Saudi Arabia \\ ${ }^{2}$ Department of Information System, Faculty of Computing and Information Technology, King Abdulaziz University, Jeddah,
} Saudi Arabia

\section{Article Info}

Received Nov 22, 2018

\section{Keyword:}

Asthma

Attack

Linear regression

Quantile regression

Accuracy

Mean absolute percentage

error

Prediction.

\begin{abstract}
Asthma is a chronic disease which concern public health over all the world. Predicting overabundant need for healthcare services is helpful for healthcare suppliers, as it allows them to adequately plan and provide the resources that deliver services easier and suitably. Several researches have linked asthma triggers with weather and environmental changes. In this study, 'linear regression model (LRM)' and 'quantile regression model (QRM)' are used to predict overabundant need for healthcare services for asthma monthly admissions in Polk, using backdated data from 2010 to 2017. Five weather variables were examined: maximum temperature, minimum temperature, precipitation level, humidity and thunderstorms. LRM and QRM models of asthma monthly admissions are fitted in two ways: the first way is the base model which using all the weather variables, and the second way is the reduced model which using a subset of these variables according to the pseudo R2. Models were cross-validated using the mean absolute percentage error and the level of accuracy. The base QRM predictive model with the 45th percentile of the distribution was the best fit and it detect utmost number of monthly asthma admissions at accuracy of $87.55 \%$, followed by base LRM with an accuracy of $87.13 \%$. The reduced LRM and QRM models give lower results with an accuracy of $86 \%$ and $85.9 \%$ respectively. The finding suggest that the weather variables are giving better results for the predictive model even if they have a low correlation relationship. The combination between weather variables and asthma, taking in temperature, precipitation level, humidity and thunderstorms can be utilized in predicting future asthma admission patients.
\end{abstract}

\section{Corresponding Author:}

Eman Alharbi,

1Department of Information System, King Abdulaziz University, Jeddah, Saudi Arabia

Email: Ealharbi0125@stu.kau.edu.sa

\section{Introduction}

Asthma is a long-standing lung disease that enrage the airways and make them narrower [1]. It has an effect on more than 355 million people in all over the world [2]. It has ranked in terms of disability adjusted life years (DALYs) as the 19th and 26th in deaths. Asthma influence is apparent not only in patients, but also in their lives and all their surrounded people, in the society in terms of lost jobs and absents days, repeated visits, hospitalizations, and death instances. Asthma cannot be cured, but taking the appropriate medication in right time can prevent many of its adverse events and complications [3].

Asthma is a disease that affected and triggered by weather. The combination between asthma morbidness and changes in weather has been notice in various studies. There is clue that the changes in temperature, humidity, air pressure, rain and wind have impact on triggering asthmatic attacks and worsening its symptoms.[4]-[7]. On-time recognition of outbreak of a disease allows quicker preparation of medical intervention and faster connection between hospitals and the patients to provide solutions and treatments. Therefore, new systems 
should be generated to assist the hospitals to control the need for treatment in a better way and prepare themselves to deal with different situations and circumstances.

Current asthma monitoring systems can have data availability tardiness far behind up to two weeks. Timely and accurate data about asthma on population and individual level are significant to put up more geographical and temporal data to asthma risk observation. The prediction of asthma trigger events based on the changes in weather conditions could lead well-timed and targeted interventions, to minimize the societal effect of asthma.

In this study, a prediction model is introduced to predict the asthma hospital admission based on the change in weather conditions at a county-scale of analysis, which is Polk in Iowa state, USA. Monthly observations of asthma emergency department visits and hospitalizations are compared with different weather variables, which are temperature, precipitation, humidity and thunderstorms in a time-series analysis. 'A linear regression model (LRM)' and 'quantile regression model (QRM)' are used. The validation of the predictive models is assessed by using the accuracy and the mean absolute percentage error measures (MAPE). This study has significant inclusion for healthcare presentation and the policies that target healthcare resources and services.

The rest of the paper is organized as the following: background in section II, literatures review in section III, methodology for asthma attack predictive model in section IV, the result and discussion in section $\mathrm{V}$, and eventually the conclusion in section VI.

\section{Background}

Environmental variables are one of the causes that severe asthma attacks and thus, the geographic location is an important factor that should be notice as the environmental variables may vary form region and another.

There are different environmental allergens and pollutants can cause an asthma attack like animal dander, smoke, and pollen. the changes in weather conditions, such as increasing or decreasing in temperature, humidity, or occurrence of thunderstorms, can trigger asthma attack also [1]. Temperature changes can cause inflammation in the airways as well. Common weather triggers that can irritate asthma symptoms include [8]:

- High temperature: when the temperature is increased, pollutants and exhaust fumes become higher and trigger asthma symptoms. Breathing in hot air can cause the airways to narrow, which causing a cough and shortness of breath [9].

- Low temperature: Cold or damp air can enter the airways and trigger a spasm, leading to asthma symptom like shortness of breath, wheezing, tightness in the chest, and coughing.

- Wind and rain: when a heavy rain occur, it can hit the grass and weed pollen, causing a break up of pollen into small pieces. These pieces are spread out in the air causing an increase in allergy which trigger asthma symptoms [10].

- Humidity: when the air is humid, the body temperature increased, causing the body to moisture. This is causing faster breathe, which can trigger asthma attack [11].

- Thunderstorms: during a storm, the wind carrying the break up pieces of pollen grains and diffusing them into the air. These pieces may entered into the lungs leading to asthma trigger [12].Provide sufficient detail to allow the work to be reproduced. Methods already published should be indicated by a reference: only relevant modifications should be described.

\section{Related Work}

There are many literatures supporting the association between changes in weather conditions and asthma morbidness. Mireku et.al [13] introduced a retrospective study to define the impacts of changes in climatic factors on increase pediatric asthma trigger. These factors include: humidity temperature, and air pressure. The relationship of asthma ED visits and changes in climactic factors was examined using time-series analysis, considering the seasonality, aeroallergen exposure and air pollution. They found that the fluctuations in humidity and temperature impact on the increasing of ED visits, while barometric pressure has no effect. Different weather variables were considered by Delamater et.al [14] who developed a study to examine the relation between weather conditions, levels of ambient air pollution and asthma hospitalizations in Los Angele. The weather variable which used were collected for all months from 2001 to 2008, and they were 
including: Particulate Matter $<10 \mu \mathrm{m}$ (PM10), Particulate Matter $<2.5 \mu \mathrm{m}$ (PM2.5), Carbon Monoxide (CO), Ozone (O3), Nitrogen Dioxide (NO2), maximum temperature, and humidity. 'Bayesian regression' models with 'temporal random effects' used to relate monthly asthma hospitalization rates to these variables. The performance of the model was evaluated using a 'goodness of fit' criterion and the predictive values. A positive correlation found between the variables PM2.5, NO2 and $\mathrm{CO}$ with asthma hospitalizations. However, different results integrated with PM10, maximum temperature and humidity, whereas no correlation found with $\mathrm{O} 3$.

Another study developed by Ayres-Sampaio et.al [15] to evaluate the relationship between several environmental variables and asthma admissions in Mainland, Portugal. They considered five environmental variables, which are: vegetation density (NDVI), relative humidity $(\mathrm{RH})$, temperature (Ta), and nitrogen dioxide (NO2). The evaluation performed over three groups which combined based on their percentage of urban cover. Ta, NO2 and NDVI makes different on the impact of asthma attack, while the other variables had no impact. They suggest that people with asthma whom existing in extremely urbanized areas are at a higher risk than other areas.

The relationship between environmental factors and asthma, including carbon monoxide, ozone and temperature were used by Soyiri et.al [16] to predict asthma future events. They predict overabundant need for healthcare services of asthma daily admissions in London. A multistage 'quantile regression models (QRM)' were adapted to a 14-lag days period of the used factors, considering seasonality. Two of the prediction models at specificity of $66 \%$ and $76 \%$, as well as sensitivity levels of $76 \%$ and $62 \%$.

Machine learning techniques have considerable effort in developing telemonitoring decision support systems for chronic disease. Finkelstein and Jeong [17] introduced a study that explore the ability of machine learning algorithms to build a prediction model for asthma by using telemonitoring data. Three classifiers were used in the evaluation, which are support vector machines, naive Bayesian, and adaptive Bayesian network. They were able to predict asthma aggravation occurring with an accuracy of $77 \%, 100 \%$, and $80 \%$, sensitivity of $80 \%, 100 \%$, and $84 \%$; and specificity of $77 \%, 100 \%$, and $80 \%$ respectively.

\section{Methodology of Asthma Attacks Prediction Model}

A set of five weather variables are considered as effective variables on triggering asthma attacks. These variables are high temperature, low temperature, precipitation level, humidity and thunderstorms. In order to build the predictive model, these variables are used as the predictor variable and the asthma admission count used as the response variable. The primary goal in this study is to evaluate the relationship between asthma attacks and these variables to find out their impact in such disease. The proposed predictive model has four layers which are: data collection layer, data pre-processing layer, data analytical layer and visualization layer. The four layers of the model are shown in Fig.1. Each of these layers are discussed below.

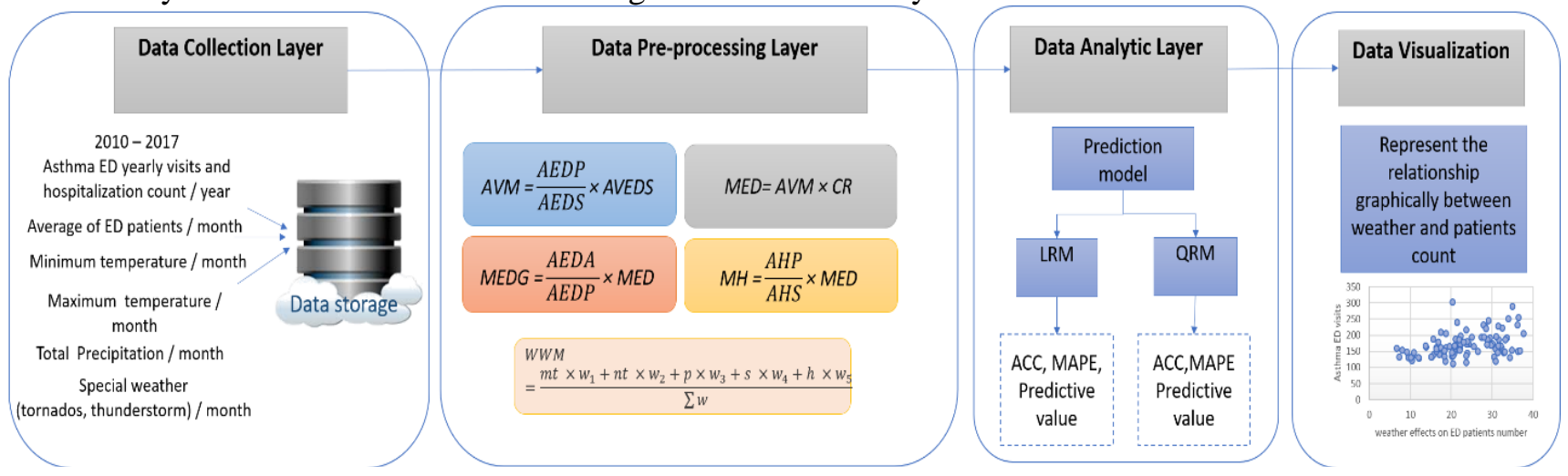

\subsection{Data Collection layer}

Figure 1. Layers of the Asthma Attacks Prediction Model

Deidentified aggregate data on Asthma emergency department ED visits and Asthma hospitalizations, with code ICD-10-CM, were captured between 2010 to 2017 for Iowa state, USA [18], [19]. These data include the number of patients seen in an ED for asthma and the number of people with asthma who were entered the hospital, respectively. Asthma hospital admission is for people who severe asthma attacks, and receive 
medical care, it is not including the outpatient people. These data can be used to find out patterns and trends of ED visits and hospitalization in different areas. We select Polk which has the highest number of patients.

This data will be compared with weather variables, which recognized as risk variables, to point out the environmental relationships with asthma triggers. These variables are: maximum temperature, minimum temperature, precipitation levels, humidity and thunderstorms. The weather dataset were collected for all months from 2010 to 2017 [20], [21], The description of the attributes which used from the dataset are placed in table 1.

Table 1. Dataset Attributes Description

\begin{tabular}{ll}
\hline Data attribute & Description \\
\hline Month & The time period per months from January to December, from 2010 to 2017 \\
Maximum temperature & Highest temperature in Fahrenheit degree recorded for the selected month and year. \\
Minimum temperature & Lowest temperature in degrees Fahrenheit recorded for the selected month and year. \\
Total Precipitation & The total of precipitation in inches recorded for the selected month and year. \\
Thunderstorms & $\begin{array}{l}\text { Shows how many tornados, ice storm and thunderstorm wind which happened in a } \\
\text { specific month. }\end{array}$ \\
Humidity & $\begin{array}{l}\text { The average of humidity recorded in a county for the selected month and year. } \\
\text { ED Visit Count }\end{array}$ \\
Thespitalization count & $\begin{array}{l}\text { The number of asthma patients who visit the emergency department } \\
\text { The of asthma inpatient hospital admissions }\end{array}$ \\
\hline
\end{tabular}

\subsection{Data Pre-processing Layer}

The pre-processing is performed over asthma dataset and the weather variables in order to create a valid time series dataset.

\section{A. Asthma Patient's data}

The collected data for asthma ED visits and hospitalization count were annual data. In order to use these data correctly we need to find out the monthly count for each of ED visits and hospitalization patients. The available monthly average of asthma ED visit's data for the whole state helped to extract the monthly data for Polk country by using the Crude Rate [18]. The 'Crude Rate (Per 10K)' is the rate of asthma patients whom entered ED or hospitalized per 10,000 population.

To find out the monthly asthma's ED visit patients data for Polk, equation (1) is used. The average monthly average of ED patients calculated by equation (2). Then, we find out the total monthly patient using equation (3).

\section{Where}

$$
\begin{gathered}
R F E D=A E D P / A E D S \\
A V M=R F E D \times A V E D S \\
M E D=A V M \times C R
\end{gathered}
$$

RFED referred to Relevant frequency of Polk ED patients,

$A E D P$ referred to annual ED patients in Polk,

$A E D S$ referred to annual ED patients in whole state,

AVM referred to Average monthly Polk data,

AVEDS referred to average ED patients of total state,

$M E D$ referred to Monthly Polk ED patient data,

$C R$ referred to Crude Rate of the year.

However, the monthly average of hospitalization data was not available in the used dataset. Therefore, we applied some mathematic calculation to extract the monthly hospitalization patients by using the monthly ED patients as a base. To do that, equations (4) and (5) are used. 


$$
\begin{gathered}
R F H=A H P / A H S \\
M H=R F H \times M E D
\end{gathered}
$$

Where

RFH referred to Relevant frequency of Polk hospitalization,

AHP referred to annual hospitalization in Polk,

AHS referred to annual hospitalization in whole state,

MH Monthly hospitalization data.

\section{B. Weather Variables Data}

Temperature, precipitation, humidity and thunderstorms can negatively affect the health of populations throughout the entire world. In general, public health impacts from climate change are predicted to include increases of ED visits and hospitalization patients. These data were collected per month from $2010-2017$. After normalizing weather dataset to 0-1 range, a special weight is given to each of these factors according to special criteria.

By studying the data and visualize the regression line between asthma patients and thunderstorm variable, it is found that the number of patients which affecting by thunderstorm whenever it occurs was small, not always but most of the time. So, a weight of $5 \%$ is given to thunderstorm variable.

Maximum and minimum temperatures are sometimes positively affecting the number of patients, when the maximum temperature became between $70-93 \mathrm{Fo}$, otherwise it is negatively affecting the number of asthma patients. Moreover, the minimum temperature is positively affecting the number of patients when it become under 46 Fo, otherwise it is negatively affecting the number of asthma patients. However, these specified degrees were not existed for long period in the dataset, therefore, a weight of $20 \%$ is given to temperature variables, $10 \%$ for maximum and $10 \%$ for minimum.

The total precipitation level, is positively affecting on patient's number in all cases. However, when the precipitation level become more than 3.5 inches, the patients number increasingly viewed, and most of the values were over 3.5 inches. Therefore, a weight of $70 \%$ is given to this variable.

The effect of the last variable, which is the humidity, found to be very small and hard to see its impact over the patient's number. Therefore, a weight of $5 \%$ is given to this variable.

Finally, the weighted mean of the variables with their weights is as shown in equation (6).

Where;

$$
W W M=\left(m t \times w_{1}+n t \times w_{2}+p \times w_{3}+s \times \times w_{4}+h \times w_{5}\right) /\left(\sum w\right)
$$

WWM is the weighted mean of weather factors, $m t$ is the maximum temperature,

$n t$ is the minimum temperature,

$p$ is the total precipitation,

$s$ is the storm and tornado,

$h$ is the humidity,

$w_{i}$ are the weights of the weather variables.

\subsection{Data Analytic Layer}

'Regression analysis' is one of the interested analysis techniques and it is used widely for analysing data. It is giving a usefulness result from the logical method of using an equation to indicate the relationship between the response variable and the predictor variables [22]. It is also attentive theoretically because of sophisticated implicit mathematics and advanced statistical theory.

In this section, 'Linear Regression Model (LRM)' and 'Quantile regression model (QRM)' are two different techniques that are employed to find out the method that gives higher prediction accuracy of asthma patients bases on given weather variables. A development of a decision tree used to generate both regression models (LRM and QRMs) of monthly asthma admissions based on the weather variables (see Fig. 2). The dataset is separated into train dataset to train the model which includes eight years of data, and validate dataset to test the model which includes the last two years of the data. The weather datasets are normalized in order to find out their weighted mean, which used as the predictor for the model. 
Two models are developed for each of the LRM and QRM, they are the base model and the reduced model. The base model is using all of the weather variables (predictor selection I in Fig.2), while the reduced model is selecting a subset of the weather variables (predictor selection II in Fig.2). The procedure of selecting a suitable subset of the weather variables is based on finding out the variables with the higher pseudo-R2, which estimated by regular linear regression. The pseudo R2 is most appropriate for comparing models of regression, which is the determination coefficient for regression and it represents the 'goodness-of-fit' statistic [23]. The reduced model includes: maximum temperature; minimum temperature and level of precipitation. The reduced model is used to predict the monthly asthma admissions and its output compared with the base model which includes all variables. The weights which used in the reduced model for these variables are as the following: $15 \%$ for maximum temperature, $15 \%$ for minimum temperature and $70 \%$ for precipitation level.

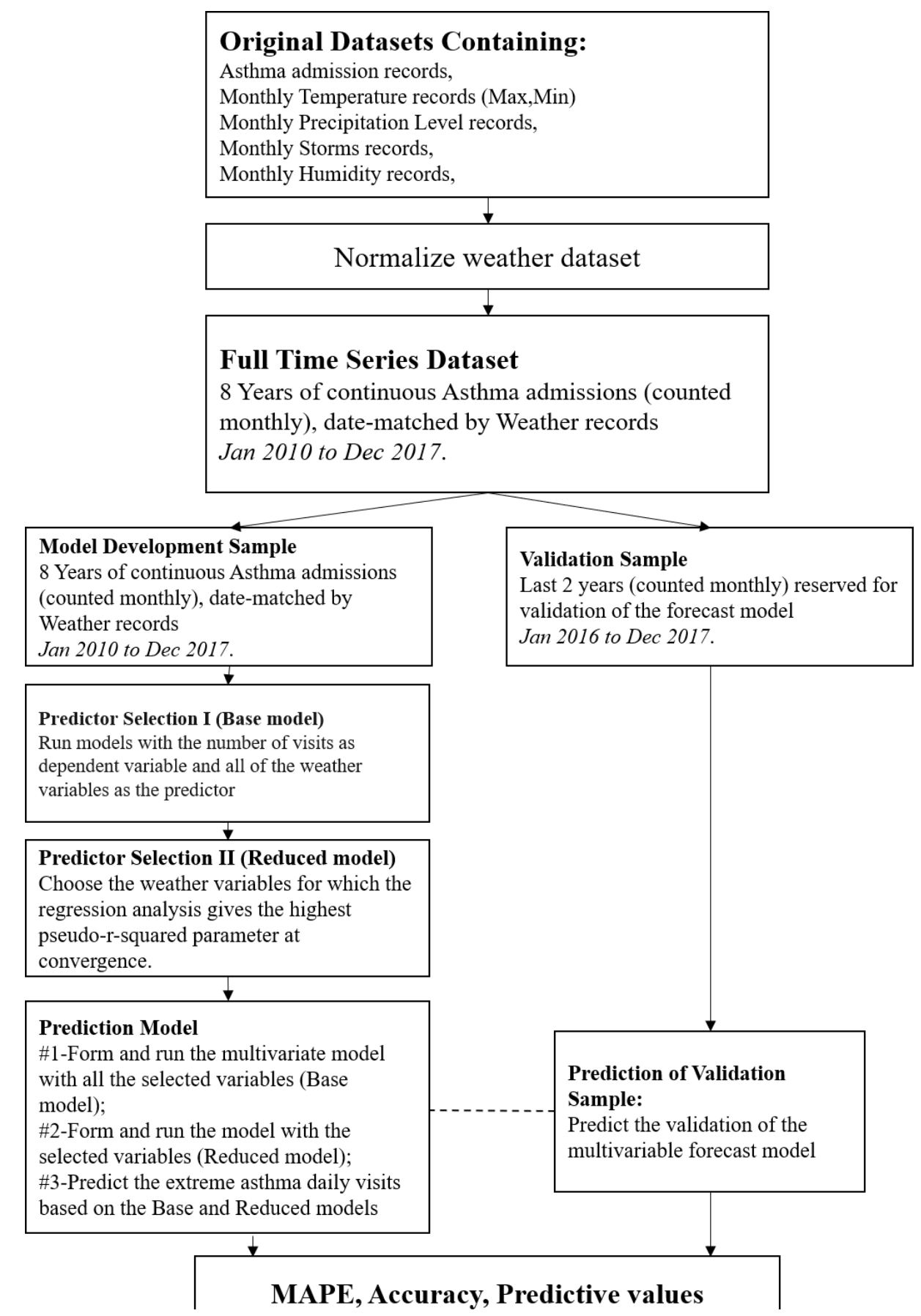

Figure 2. Decision tree for LRM and QRM prediction models 


\section{A. Linear Regression Model (LRM)}

The combination of asthma attacks and weather variables for each month in the period of 2010-2017 is evaluated by a LR analysis. The response variable is the asthma patient's admission rate per 10000 inhabitants in Polk country, and the weighted mean for the five weather variables (Max temp, Min temp, precipitation, humidity and thunderstorms) is the predictor variable.

LRM is built for both of our predictor selection (base and reduced model) to find the best fit line and thus determine the corresponding slope and y-intercept with the total patient's number (hospitalization and ED visits), see Fig.3

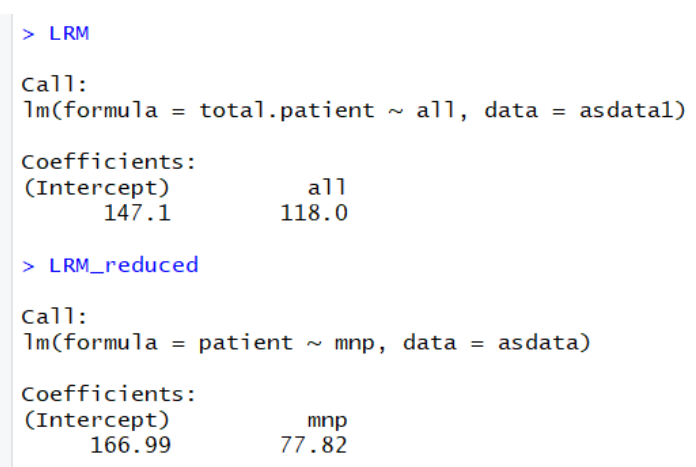

Figure 3. The Coefficient and Intercept of LRM for base and reduced models

\section{B. Quantile Regression Models (QRMs)}

QRMs are a preferable method for modelling and predicting cases as they are providing finer relationship between a response distribution and predictor variables for specific quantiles [24]. The most used quantiles in the literatures are $0.4,0.5$ or 0.9 . However, to select the best quantile to use in asthma attacks prediction model, we test the QRM with nine quantiles which are: $0.15,0.25,0.35,0.45,0.55,0.65,0.75,0.85$ and 0.95 . QRM is developed for both base and reduced model to find the best fitting regression line and thus define the identical intercept and slope with the total patient's number, see Fig.4.

For the predicted monthly asthma attacks, the QRM can be calculated by using equation (7):

$$
y_{i}=\beta_{0}^{p}+\beta_{1}^{p} x_{i}+\varepsilon_{i}^{p}
$$

Where:

$y_{i}$ is 'asthma hospital admissions' for a given month, $i$

$\beta_{0}^{p}$ is' a constant,

$\beta_{1}^{p}$ is 'a coefficient of exposure term,

$x_{i}$ is 'the exposure term,

$p$ is 'the quantile,

$\varepsilon_{i}^{p}$ is 'the error term. 


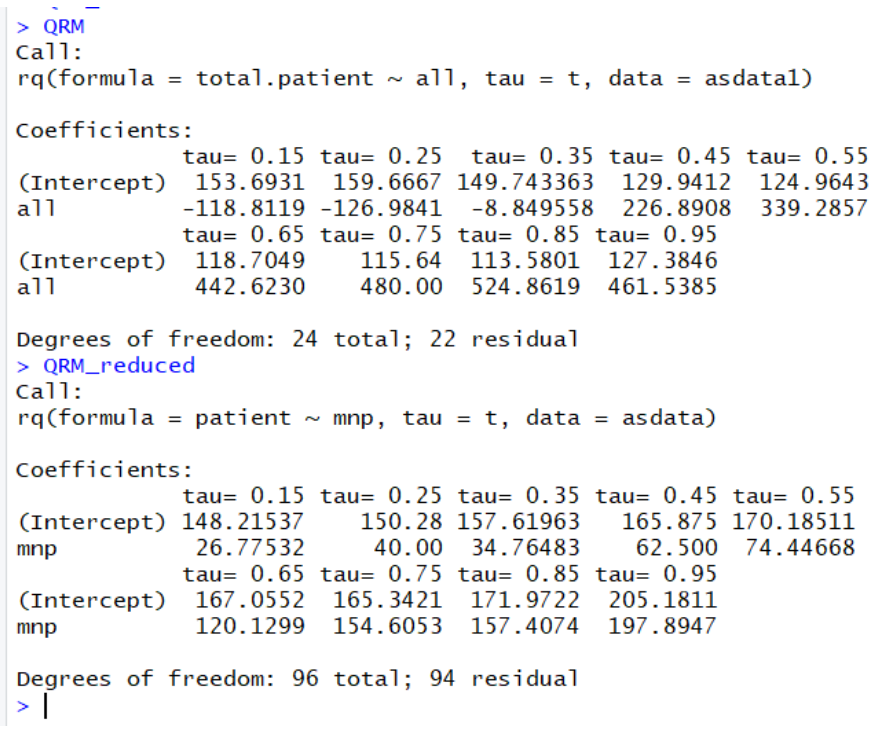

Figure 4. The coefficient and interception of QRM for base and reduced models

\subsection{Data Visualization Layer}

In this layer, some plots for the analysed dataset are shown. Fig.5 represents the diagram of time serious plot for the total asthma patient's admission, including ED visits and hospitalization patients in the duration between 2010 and 2017. We can observe from this visualization that there are seasonality of the asthma admissions and there are two peaks in most of the years. From Fig.5, we can observe that the peaks occur during the summer and winter quarters, this is could be due to the changes in temperature and the frequent rain.

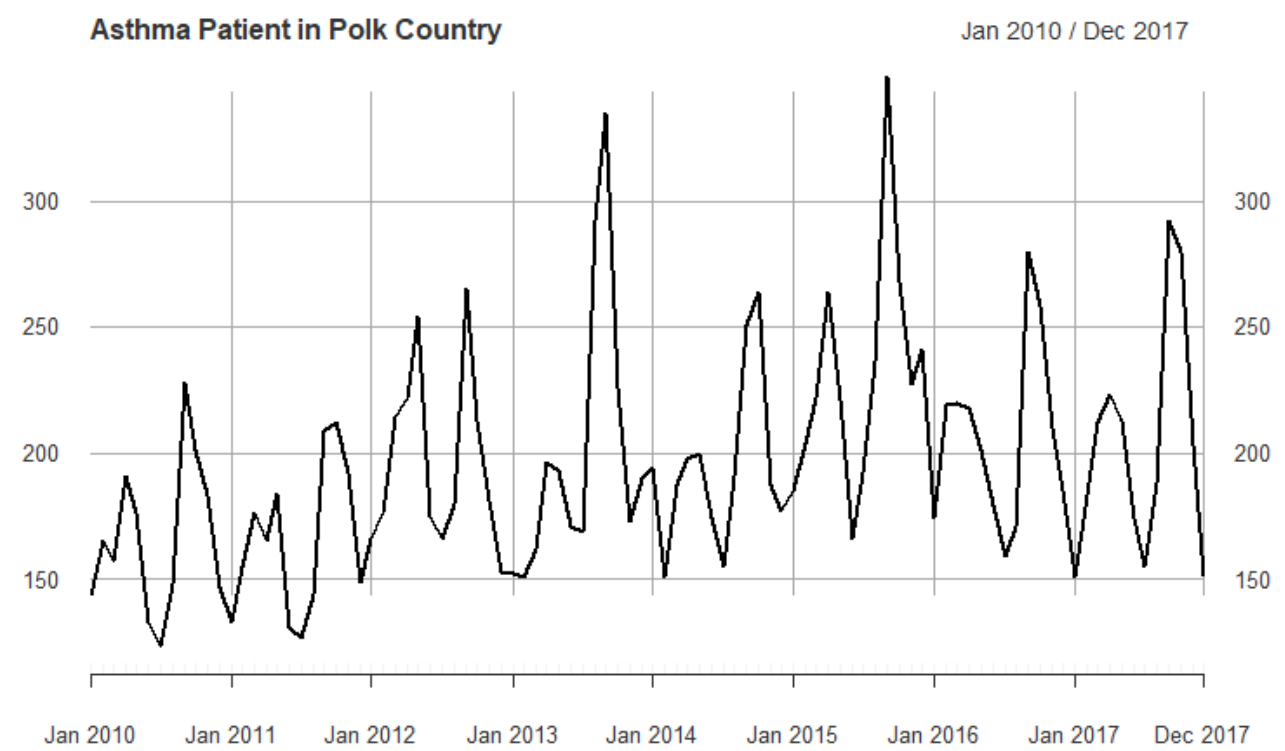

Figure 5. Total Asthma Patients (ED visits and hospitalization) in Polk, Iowa.

The weather variables are computed by using their weights, then a scatter plot is used to visualize the relationship between their weighted mean and the total asthma's patients as shown in Fig 6. The visualized line is representing the positive correlation between both variables with 0.21 pseudo R2. In Fig.7, each line represents the regression line of specific quantile starting from bottom line with quantile 0.15 to the top line with quantile 0.95 . 
Linear Regrission of Asthma and Weather

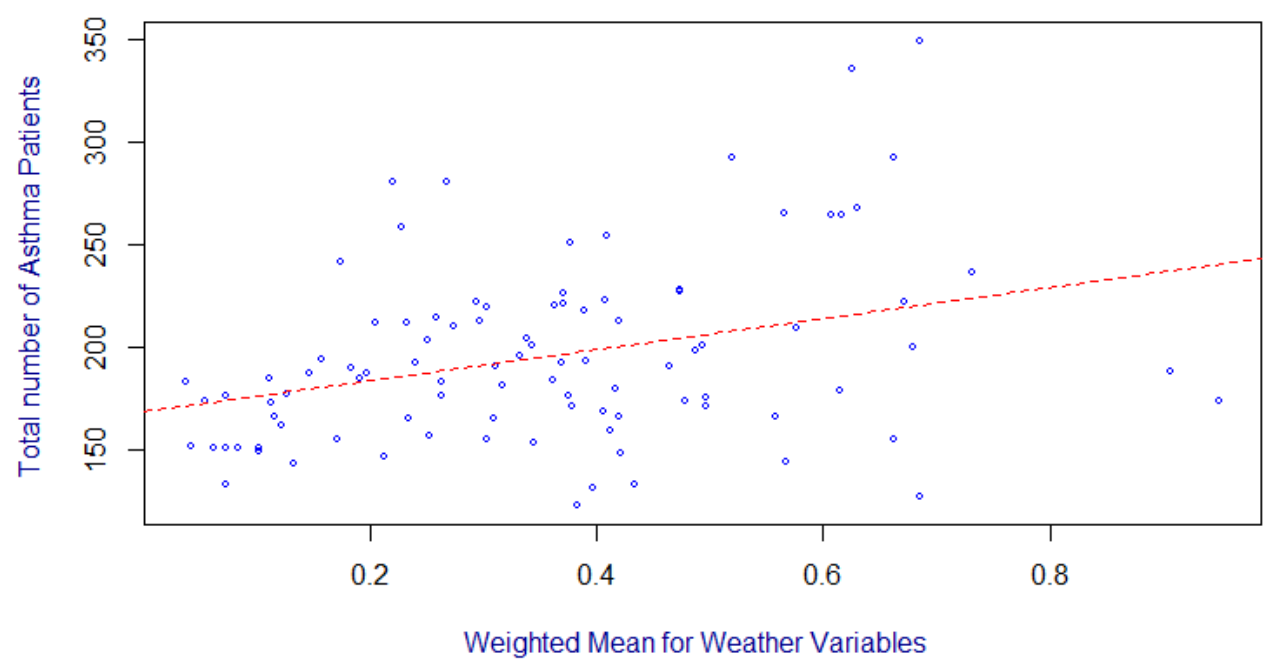

Figure 6. The LRM Scatter Plot between Asthma's Patient and weighted mean of weather

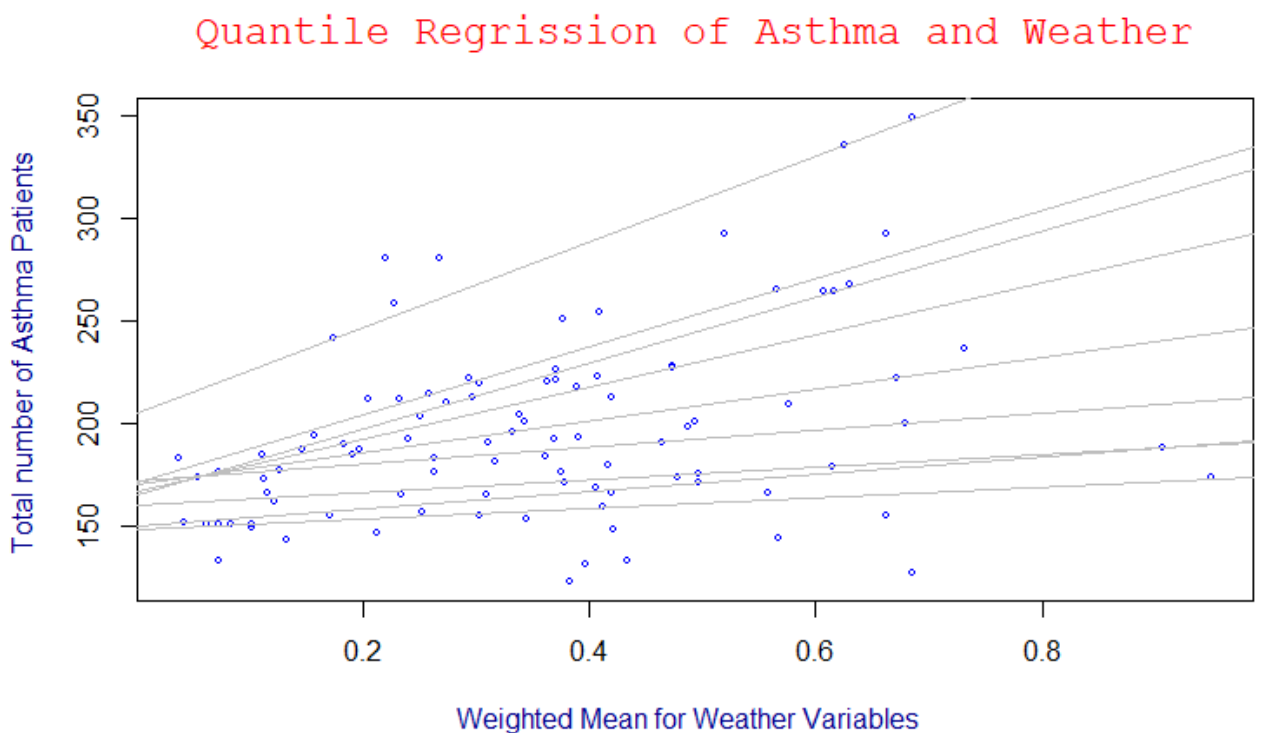

Figure 7. The QRM Scatter Plot between Asthma's Patient and Weighted Mean of Weather

\section{Results and Discussion}

In this study, we used $\mathrm{R}$ to build our models. $\mathrm{R}$ is a programming language used for statistical analysis and graphics, it is offered as open source [25]. It consists of a core (that already includes a number of packages) and contributed packages programmed by user around the world. The main package which used by this study is quantreg [26],

The predictive validity of LRM and QRMs models are compared using two performance measures: the accuracy and mean absolute percentage error (MAPE) measures. LRM and QRM has been trained for both predictor selection: the base and reduced models with the data from 2010 to 2017. The validation sample, which is from 2016 to 2017, tested for both LRM and QRM for base and reduced models, and the results are placed in Table 2 and 3. The accuracy for LRM's base model (i.e. uses all the weather variables) found to be $87.13 \%$ with MAPE of 0.14 . While in LRM's reduced model, (i.e. uses some selective weather variables), the accuracy decreased to be $86 \%$ with MAPE of 0.16 . 
In QRM's base model, the best quantile which gives the highest accuracy is 0.45 , and the accuracy found to be $87.55 \%$ with MAPE of 0.14 . While in QRM's reduced model, the best quantile is 0.55 and the accuracy is $85.97 \%$ with MAPE of 0.16 .

Table 2. MAPE and Accuracy of LRM and QRM for Base Model

\begin{tabular}{|c|c|c|c|c|}
\hline \multicolumn{2}{|c|}{ LRM } & \multicolumn{3}{|c|}{ QRM } \\
\hline MAPE & Accuracy & Quantile & MAPE & Accuracy \\
\hline \multirow[t]{9}{*}{0.146} & $87.13 \%$ & $\mathrm{P}=0.15$ & 0.166 & $83.13 \%$ \\
\hline & & $\mathrm{P}=0.25$ & 0.144 & $86.0 \%$ \\
\hline & & $\mathrm{P}=0.35$ & 0.144 & $86.0 \%$ \\
\hline & & $\mathrm{P}=0.45$ & 0.144 & $87.55 \%$ \\
\hline & & $\mathrm{P}=0.55$ & 0.145 & $87.51 \%$ \\
\hline & & $P=0.65$ & 0.185 & $87.05 \%$ \\
\hline & & $P=0.75$ & 0.193 & $86.78 \%$ \\
\hline & & $\mathrm{P}=0.85$ & 0.216 & $85.55 \%$ \\
\hline & & $\mathrm{P}=0.95$ & 0.238 & $83.72 \%$ \\
\hline
\end{tabular}

Table 3. MAPE and Accuracy of LRM and QRM for Reduced Model

\begin{tabular}{|c|c|c|c|c|}
\hline \multicolumn{2}{|c|}{ LRM } & \multicolumn{3}{|c|}{ QRM } \\
\hline MAPE & Accuracy & Quantile & MAPE & Accuracy \\
\hline \multirow[t]{9}{*}{0.160} & $86.09 \%$ & $\mathrm{P}=0.15$ & 0.209 & $79.17 \%$ \\
\hline & & $\mathrm{P}=0.25$ & 0.183 & $81.95 \%$ \\
\hline & & $\mathrm{P}=0.35$ & 0.164 & $84.12 \%$ \\
\hline & & $\mathrm{P}=0.45$ & 0.157 & $85.58 \%$ \\
\hline & & $\mathrm{P}=0.55$ & 0.160 & $85.97 \%$ \\
\hline & & $\mathrm{P}=0.65$ & 0.174 & $85.67 \%$ \\
\hline & & $\mathrm{P}=0.75$ & 0.206 & $84.42 \%$ \\
\hline & & $\mathrm{P}=0.85$ & 0.231 & $83.24 \%$ \\
\hline & & $\mathrm{P}=0.95$ & 0.314 & $78.69 \%$ \\
\hline
\end{tabular}

Predicting overabundant need for healthcare services is helpful to healthcare suppliers, as it allows them to adequately plan and provide the resources that deliver services easier and suitably. In this study, two methods for implementing prediction models for expected number of asthma patients in Polk country, Iowa is designed. The LRM and QRM base models can predict these admissions at accuracy of $87.13 \%$ and $87.55 \%$ respectively, when using all of the weather variables which applied to this study, (i.e. temperature, precipitation, humidity and storms). However, the existence of low correlation between asthma admission count and some of weather variables did not give a reason to drop these variables from our predictive model, which are improved by testing the reduced model and the accuracy reduced to be $86 \%$ and $85.97 \%$ in LRM and QRM respectively.

The publications on prediction propose that there is no standardised method to predict any special cases, and they suggests a supplement of several methods [27], [28]. QRM presents an option for predicting asthma hospital admissions [29], [30]. In this study, the asthma patient's admission is notionally realized by the 45th percentile of the distribution in QRM model.

In this study, we specified five variables and designate a base prediction model with these variables. However, we tried to find a simpler method of predicting the asthma attacks, by reducing the weather variables from five to three variables based on the pseudo R2. This reduced model, did not provides better estimation than the 
base model. Therefore, it is better to use more weather variables which are related to the asthma attacks trigger even if that variable has low impact on influencing asthma symptoms. The predictors used in the model reconfirm the combination of asthma and different weather variables such as temperature, precipitation, humidity and storms. This relationship is consistent with the literature discussed.

\section{Conclusions}

Overabundant need for healthcare services is a great challenge to any healthcare service suppliers, but the capability to predict cases is a powerful research area. In this study, a prediction model is developed to predict the asthma admission based on the changes in weather, including maximum and minimum temperatures, precipitation level, humidity and thunderstorms. LRM and QRMs are used to build the predictive model. These models are compared in two ways: the base model which includes all the weather variables and the reduced model which includes selected subset of the weather variables. The selection process is done based on the existence of high correlation between the selected weather variable and the asthma counts (pseudo R2). The base LRM and QRM successes in predicting the number of monthly asthma admissions at accuracy of $87.13 \%$ and $87.55 \%$ respectively. We found that the quantile was better and gives best accuracy and lowest MAPE at the 45th percentile of the distribution when using the base model. The accuracy of LRM and QRM was decreased by using the reduced model, it was $86 \%$ and $85.95 \%$ respectively. The findings suggest that having many weather variables that have an impact on asthma, even if they don't correlated strongly with the asthma counts, may positively effective the prediction model and gives better results. The results reaffirm the known associations between asthma and temperature, precipitation level, humidity and storms. As a future work, we plan to build an application that can be used by hospitals that able to acquire weather data and find out the changes in the variables that affect asthma triggers, then predict the number of asthma patients in order to prepare the required facilities and medicine.

\section{References}

[1] "Asthma | National Heart, Lung, and Blood Institute (NHLBI)." [Online]. Available: https://www.nhlbi.nih.gov/health-topics/asthma. [Accessed: 15-Oct-2018].

[2] "The Global Asthma Report 2018." [Online]. Available: http://globalasthmareport.org/. [Accessed: 01-Dec-2018].

[3] N. A. E. and P. P. Asthma Third Expert Panel on the Diagnosis and Management of, Expert Panel Report 3: Guidelines for the Diagnosis and Management of Asthma. National Heart, Lung, and Blood Institute (US), 2007.

[4] M. J. Carey and I. Cordon, "Asthma and climatic conditions: experience from Bermuda, an isolated island community.," Br. Med. J. Clin. Res. Ed, vol. 293, no. 6551, pp. 843-844, Oct. 1986.

[5] J. H. Silber, "Forecasting asthmatic wheezing using temperature velocity," Pediatr. Emerg. Care, vol. 3, no. 1, pp. 13-17, Mar. 1987.

[6] A. S. Khot, R. Burn, N. J. Evans, W. Lenney, and J. N. Storr, "Biometeorological triggers in childhood asthma.," Clin. Allergy, vol. 18, no. 4, pp. 351-358, 1988.

[7] Y. Zhang et al., "Effects of Meteorological Factors on Daily Hospital Admissions for Asthma in Adults: A Time-Series Analysis," PLOS ONE, vol. 9, no. 7, p. e102475, Jul. 2014.

[8] "How Weather Affects Asthma," EverydayHealth.com. [Online]. Available: https://www.everydayhealth.com/asthma/how-weather-affects-asthma.aspx. [Accessed: 13-Oct-2018].

[9] "Weather as an asthma trigger," Asthma UK. [Online]. Available: https://www.asthma.org.uk/advice/triggers/weather/. [Accessed: 01-Dec-2018].

[10] A. C. Services, "How Does Rain Affect Pollen Levels?," Asthma and Allergy Foundation of America. [Online]. Available: https://community.aafa.org/blog/how-does-rain-affect-pollen-levels. [Accessed: 01-Dec2018].

[11] "3 Ways Humidity Affects Asthma | Asthma and Allergy Foundation of America." [Online]. Available: https://community.aafa.org/blog/3-ways-humidity-affects-asthma. [Accessed: 01-Dec-2018].

[12] A. C. Services, "Thunderstorms and Asthma: An Unexpected Connection," Asthma and Allergy Foundation of America. [Online]. Available: https://community.aafa.org/blog/thunderstorms-and-asthma-anunexpected-connection. [Accessed: 01-Dec-2018]. 
[13] N. Mireku, Y. Wang, J. Ager, R. C. Reddy, and A. P. Baptist, "Changes in weather and the effects on pediatric asthma exacerbations," Ann. Allergy. Asthma. Immunol., vol. 103, no. 3, pp. 220-224, Sep. 2009.

[14] P. L. Delamater, A. O. Finley, and S. Banerjee, "An analysis of asthma hospitalizations, air pollution, and weather conditions in Los Angeles County, California," Sci. Total Environ., vol. 425, pp. 110-118, May 2012.

[15] D. Ayres-Sampaio, A. C. Teodoro, N. Sillero, C. Santos, J. Fonseca, and A. Freitas, "An investigation of the environmental determinants of asthma hospitalizations: An applied spatial approach," Appl. Geogr., vol. 47, pp. 10-19, Feb. 2014.

[16] I. N. Soyiri, D. D. Reidpath, and C. Sarran, "Forecasting peak asthma admissions in London: an application of quantile regression models," Int. J. Biometeorol. Heidelb., vol. 57, no. 4, pp. 569-78, Jul. 2013.

[17] J. Finkelstein and I. cheol Jeong, "Machine learning approaches to personalize early prediction of asthma exacerbations," Ann. N. Y. Acad. Sci., vol. 1387, no. 1, pp. 153-165, Jan. 2017.

[18] "Asthma Emergency Department Visits." [Online]. Available: https://tracking.idph.iowa.gov/Health/Asthma/Asthma-ED-Visits. [Accessed: 02-Nov-2018].

[19] "Hospitalization Data." [Online]. Available: https://tracking.idph.iowa.gov/Health/Asthma/hospitalizations-asthma. [Accessed: 02-Nov-2018].

[20] "Temperature and Precipitation Data." [Online]. Available: https://tracking.idph.iowa.gov/Environment/Climate/Temperature-and-Precipitation-Data. [Accessed: $02-$ Nov-2018].

[21] "Storm Events Database - Search Page | National Centers for Environmental Information." [Online]. Available: https://www.ncdc.noaa.gov/stormevents/choosedates.jsp?statefips=19\%2CIOWA. [Accessed: 06Nov-2018].

[22] A. C. Rencher and G. B. Schaalje, Linear models in statistics, 2nd ed. Hoboken, N.J: WileyInterscience, 2008.

[23] J. Zietz, E. N. Zietz, and G. S. Sirmans, "Determinants of House Prices: A Quantile Regression Approach,” J. Real Estate Finance Econ., vol. 37, no. 4, pp. 317-333, Nov. 2008.

[24] S. M. Barbosa, "Quantile trends in Baltic sea level," Geophys. Res. Lett., vol. 35, no. 22, Nov. 2008.

[25] "R: The R Project for Statistical Computing." [Online]. Available: https://www.r-project.org/. [Accessed: 02-Dec-2018].

[26] R. Koenker, S. P. (Contributions to C. Q. code), P. T. N. (Contributions to S. Q. code), A. Z. (Contributions to dynrq code essentially identical to his dynlm code), P. G. (Contributions to nlrq code), and B. D. R. (Initial (2001) R. port from S. (to my everlasting shame--how could I. have been so slow to adopt R. and for numerous other suggestions and useful advice), quantreg: Quantile Regression. 2018.

[27] J. S. Armstrong, "Principles of Forecasting: A Handbook for Researchers and Practitioners," p. 862.

[28] R. Fildes, K. Nikolopoulos, S. F. Crone, and A. A. Syntetos, "Forecasting and operational research: a review," J. Oper. Res. Soc., vol. 59, no. 9, pp. 1150-1172, Sep. 2008.

[29] I. N. Soyiri and D. D. Reidpath, "Evolving forecasting classifications and applications in health forecasting,” Int. J. Gen. Med., vol. 5, pp. 381-389, 2012.

[30] I. N. Soyiri and D. D. Reidpath, "An overview of health forecasting," Environ. Health Prev. Med., vol. 18, no. 1, pp. 1-9, Jan. 2013.

[31] K. L. Ebi and J. K. Schmier, "A stitch in time: improving public health early warning systems for extreme weather events," Epidemiol. Rev., vol. 27, pp. 115-121, 2005.

[32] L. Breiman, "Statistical Modeling: The Two Cultures," Stat. Sci., vol. 16, no. 3, pp. 199-215, 2001. 